\title{
Exploring the recollective experience during autobiographical memory retrieval in amnestic mild cognitive impairment
}

\author{
MUIREANN IRISH, ${ }^{1,2}$ BRIAN A. LAWLOR, ${ }^{1}$ SHANE M. O'MARA, ${ }^{2}$ AND ROBERT F. COEN ${ }^{1}$ \\ ${ }^{1}$ Mercer's Institute for Research on Aging, St. James's Hospital, Dublin 8, Ireland \\ ${ }^{2}$ Trinity College Institute of Neuroscience, Trinity College, Dublin 2, Ireland
}

(Received September 7, 2009; Final Revision January 23, 2010; AcCePted January 28, 2010)

\begin{abstract}
Autonoetic consciousness refers to the ability to mentally transport oneself back in subjective time to relive elements of, or all, of a past event, and is compromised in the early stages of Alzheimer's disease (AD). Here, we investigate autobiographical memory (ABM) and the recollective experience in amnestic mild cognitive impairment (aMCI). aMCI participants exhibited significant deficits compared with healthy elderly controls for both personal semantic and event detail components of ABM. These decrements were evident across all life epochs for episodic recall. Recall of an event that occurred 1 week previously, was tested in the same spatiotemporal context, and provided the greatest group dissociation, with elderly controls benefitting from a context-dependent memory effect. This reinstantiation of context did not ameliorate the anterograde deficits in the aMCI cohort, nor did it facilitate the mental reliving of these memories for either participant group. Whereas reliving judgments were comparable in both groups, aMCI participants exhibited a compromised capacity to generate vivid, self-referential visual imagery and to re-experience the original emotion of events. These contextual and experiential deficits extended beyond recently encountered events into remote epochs, and suggest a greater level of ABM impairment in aMCI than previously assumed. (JINS, 2010, 16, 546-555.)
\end{abstract}

Keywords: Aging, Autonoetic consciousness, Context-dependent memory, Eidetic imagery, Emotion, Episodic memory

\section{INTRODUCTION}

Mild cognitive impairment (MCI) refers to individuals who show objective decrements in cognitive functioning, often at an intermediate level to normal aging and Alzheimer's disease (AD), yet not to a sufficient degree for dementia criteria to be fulfilled (Arnaiz \& Almkvist, 2003). There is considerable debate regarding the utility of $\mathrm{MCI}$ as a diagnostic entity (Petersen \& Morris, 2005; Pike \& Savage, 2008), as some studies have shown evidence in favor of progression to dementia, most commonly AD (Fischer et al., 2007), in contrast with studies demonstrating outcomes including depression (Aggarwal, Wilson, Beck, Bienias, \& Bennett, 2005), progression to other forms of dementia such as vascular dementia (Visser, Scheltens, \& Verhey, 2005), stable MCI (Ganguli, Dodge, Shen, \& DeKosky, 2004), and apparent improvement (Luis et al., 2004). This heterogeneity of MCI

Correspondence and reprint requests to: Muireann Irish, Mercer's Institute for Research on Aging, Hospital 4, St. James's Hospital, Dublin 8, Ireland.E-mail: irishmu@tcd.ie or rcoen@stjames.ie is manifested in varying cognitive profiles and underlying etiologies (Petersen \& Morris, 2005; Petersen et al., 2001), and whereas samples of MCI individuals may include people who will progress to $\mathrm{AD}$, they invariably will include people who will not (Pike \& Savage, 2008). Here, we focus on the subtype of amnestic MCI (aMCI) which is associated with greater risk of developing clinically probable $\mathrm{AD}$ (Gauthier et al., 2006).

Neuropsychological assessments tend to focus on memory as the main region of interest, with aMCI individuals typically exhibiting deficits on tests of anterograde memory at a level that is intermediate to normal aging and AD (Perri, Carlesimo, Serra, \& Caltagirone, 2005). Murphy, Troyer, Levine, and Moscovitch (2008) note that such behavioral deficits are reflected in the neuroanatomical changes associated with aMCI, with pathology in the hippocampus and entorhinal cortex at a transitional level to normal aging and $\mathrm{AD}$ (Masdeu, Zubieta, \& Arbizu, 2005; Pennanen et al., 2004). Delacourte et al. (1999) note that episodic memory is a reliable neuropsychological marker of $\mathrm{AD}$, and tasks assessing episodic memory may be particularly useful in the detection 
of at-risk individuals (Tabert et al., 2006). One branch of episodic memory which has received little attention in MCI is remote autobiographical memory (ABM).

ABM refers to the recollection of personal facts and episodes from one's past and can persist across various temporal categories, retaining knowledge of the self at different levels of abstraction (Conway, Pleydell-Pearce, Whitecross, \& Sharpe, 2002). Several systems are implicated in ABM (Rubin, 2005), including memory for details of the event (Moscovitch, Yaschyshyn, Ziegler, \& Nadel, 1999), a sensory image often in several modalities (Brewer, 1996; Conway, 2001), a spatial sense of the location of actors and objects in the event, and self-referential (Addis, Moscovitch, Crawley, \& McAndrews, 2004) and emotional (Piefke, Weiss, Zilles, Markowitsch, \& Fink, 2003) connotations. Retrieval of episodic details is compromised in healthy aging, leading to a greater production of semantic details lacking spatiotemporal specificity (Levine, Svoboda, Hay, Winocur, \& Moscovitch, 2002; Piolino, Desgranges, Benali, \& Eustache, 2002). A similar pattern is evident in aMCI, with participants producing reduced episodic details and increased levels of semantic details (Murphy et al., 2008). However, more recently, Leyhe, Muller, Milian, Eschweiler, and Saur (2009) documented impairments in both personal semantics and autobiographical episodes in aMCI.

Enmeshed within ABM is the phenomenon of autonoetic consciousness, which mediates the capacity for mental time travel (Tulving, 2001) facilitating a rich sense of recollection of past events. The recollective experience is disrupted in AD with a significant reduction in "Remember" judgments on the Remember/Know paradigm (R/K) (Piolino et al., 2003; Rauchs et al., 2007). More recently, AD patients have been shown to exhibit a compromised capacity to mentally relive past events, as well as phenomenological changes such as impoverished visual imagery and dampening of emotional re-experiencing (Irish et al., in press).

To date, it has not been documented whether the accompanying recollective experience of ABM is significantly disrupted in aMCI. The objectives of the present study were two-fold. First, we wished to examine potential differential impairments in personal semantic and episodic ABM in aMCI. Second, we wished to explore the phenomenon of autonoetic consciousness during $\mathrm{ABM}$ recall in aMCI, with a view to uncovering which elements of $\mathrm{ABM}$ retrieval are important for the recollective experience. Given that significant deficits in episodic ABM have been demonstrated in aMCI (Murphy et al., 2008), we hypothesized that our participants would show impaired recall of contextual episodic details, manifesting in a compromised capacity to relive these events.

\section{METHOD}

\section{Participants}

Participants consisted of 18 healthy elderly controls (EC) and 16 amnestic MCI individuals. MCI participants were recruited through the Mercer's Institute for Research on Aging
(MIRA), where they attended for diagnosis and follow-up. Amnestic MCI (aMCI) was defined according to the Winblad et al. (2004) criteria, whereby (i) the person was neither normal nor demented, (ii) there was objective evidence of cognitive deterioration specifically in memory (test scores 1.5 standard deviations < age-and education-matched normative means) corroborated by subjective report of decline by self and/or informant, and (iii) activities of daily living were preserved and complex instrumental functions were either intact or minimally impaired. A multi-disciplinary team arrived at the diagnosis of aMCI in line with the MCI Working Group of the European Consortium on Alzheimer's disease criteria (Portet et al., 2006), emphasizing the preserved functional capacity of the individual. According to Petersen and Morris' (2005) taxonomy of aMCI, the majority of participants in the sample $(n=13)$ represented multidomain aMCI as they exhibited deficits in memory and executive functioning (see Table 1), whereas the remaining 3 participants' deficits resided exclusively within the domain of memory (single-domain aMCI).

\section{Cognitive screening}

Exclusion criteria for controls included scoring $<27$ on the Mini-Mental State Examination (MMSE; Folstein, Folstein, \& McHugh, 1975), <8 on the Clock Drawing Test (CDT; Manos \& Wu, 1994; 10-point scoring system). The Geriatric Depression Scale 15-item version (GDS-15; Sheikh \& Yesavage, 1986) was used to determine incidences of pervasive depression with a cut-off score of $>7$ chosen to maximise specificity (see Herrmann et al., 1996). The National Adult Reading Test (NART; Nelson \& Willison, 1991) was included as a measure of premorbid IQ, and the Instrumental Activities of Daily Living scale (IADL; Lawton \& Brody, 1969) was administered to ensure that participants did not show evidence of functional decline (score $<8$ on IADL). Further exclusion criteria for both groups included history of psychiatric illness or alcohol abuse, or significant head injury. Participants were also assessed across several tasks of executive function including digit and spatial span (WMS-III, Wechsler, 1997), letter and category fluency, Trail Making Test (Reitan, 1958), and the Stroop test (Trenerry, Crosson, DeBoe, \& Leber, 1989).

Ethical approval was obtained from the St. James's Hospital and Adelaide and Meath Research Ethics Committee and all participants gave informed consent before testing in accordance with the Declaration of Helsinki (1991).

\section{MATERIALS AND PROCEDURE}

The Episodic Autobiographical Memory Interview (EAMI; Irish, Lawlor, O'Mara, \& Coen, 2008) is a semi-structured interview that assesses $\mathrm{ABM}$ recall across the lifespan from Childhood (0-15 years), Early Adulthood (16-30 years), Middle Adulthood (31-45 years), Later Adulthood (46 up to 5 years ago), and Recent memory (in the last 5 years). The EAMI assesses recall of Personal Semantics (PS), autobiographical 
Table 1. Demographics and neuropsychological test performance (mean $\pm S D$ ) for EC and aMCI individuals

\begin{tabular}{|c|c|c|c|c|}
\hline & Assessment & EC group $(n=18)$ & aMCI group $(n=16)$ & Group effect \\
\hline \multirow[t]{3}{*}{ Demographics } & Age & $76.0(4.3)$ & $71.8(6.8)$ & $*$ \\
\hline & Education & $14.0(3.1)$ & $13.8(4.7)$ & $\mathrm{n} / \mathrm{s}$ \\
\hline & Gender & $4 \mathrm{M}: 14 \mathrm{~F}$ & $10 \mathrm{M}: 6 \mathrm{~F}$ & $*$ \\
\hline \multirow[t]{5}{*}{ Screening } & MMSE“7s” & $28.9(.9)$ & $26.4(1.7)$ & $* *$ \\
\hline & MMSE "dlrow" & $29.3(.8)$ & $26.6(2.2)$ & $* *$ \\
\hline & NART IQ & $116.2(6.3)$ & $111.8(7.8)$ & $\mathrm{n} / \mathrm{s}$ \\
\hline & CDT & $9.3(1.0)$ & $7.9(2.0)$ & $*$ \\
\hline & GDS & $1.3(1.5)$ & $1.7(1.3)$ & $\mathrm{n} / \mathrm{s}$ \\
\hline \multirow[t]{6}{*}{ Executive Function } & Digit Span & $17.8(3.6)$ & $15.7(5.1)$ & $\mathrm{n} / \mathrm{s}$ \\
\hline & Spatial Span & $12.4(2.5)$ & $10.4(2.4)$ & $*$ \\
\hline & Letter fluency & $44.3(15.1)$ & $37.7(10.7)$ & $\mathrm{n} / \mathrm{s}$ \\
\hline & Category fluency & $43.7(9.7)$ & $31.2(5.9)$ & $* *$ \\
\hline & Trail Making Test (B-A) & $57.4(32.1)$ & $97.4(45.4)$ & $*$ \\
\hline & Stroop Test (percentiles) & $28.6(27.8)$ & $10.2(13.3)$ & $*$ \\
\hline \multirow[t]{2}{*}{$\mathrm{ABM}$ retrieval } & EAMI Total PS & $65.4(2.1)$ & $58.3(5.2)$ & $* *$ \\
\hline & EAMI Event Details & $31.5(3.4)$ & $20.9(3.9)$ & $* *$ \\
\hline
\end{tabular}

Note. Group effect determined by multivariate analysis of variance (MANOVA) for demographic variables and tests of executive function except the Stroop test which was non-normally distributed. Mann-Whitney $U$ tests for screening variables as distribution of variables were non-normal, except for Gender where $\chi^{2}$ statistics were used; ** $p<.001 ; * p<.05$.

$\mathrm{PS}=$ Personal Semantics; $\mathrm{n} / \mathrm{s}=$ nonsignificant

event details and the autonoetic recollective experience across each epoch.

\section{Personal Semantics}

The Personal Semantic subscale is adopted from other measures of ABM (e.g., Kopelman, Wilson, \& Baddeley, 1989; Piolino et al., 2002) requiring participants to recall information from three discrete categories of information; (i) Names of people, (ii) Daily Living, and (iii) Important Dates. Participants were encouraged to provide full names of three people and their relationship to each person (Score 1 for each full name and 1 point for relationship = maximum of 6 points), information relating to Daily Living (1 point for Name of institution/association, 1 point for location, 1 point for nature of work/study/hobby, 1 point for how participant travelled there $=$ maximum of 4 points), and a specific date for an important event ( 1 point for date, 1 point for month, 1 point for year, and 1 point for location of event $=$ maximum of 4 points). Repetitions across epochs were awarded no points.

\section{Autobiographical Event Recall}

On the event subscale, participants were instructed to recall in detail one event that occurred during each life period according to the following instructions, "I would like you to describe out loud and with as much detail as possible, an event that occurred during this time period that stands out for you." If participants could not spontaneously bring an event to mind, cues specific to the epoch under investigation were provided (e.g., "your wedding day," "a trip you took," "a family event"). Participants were encouraged to engage in a free recall of the event, following which the experimenter probed for further details using seven phenomenological categories, modified from the Event Details Checklist of Moscovitch et al. (1999). The seven categories assessed Event detail, Temporal Specificity, Sensory/Perceptual Details, Spatial Specificity, Emotion, Implication of Event, and Thoughts. Scoring for events took into account the specificity of the event (i.e., whether it was a unique once-off event or a repeated occurrence) with a maximum of 7 points awarded for each event (see Irish et al., in press, for full scoring protocol). Contextual details were scored as 0.5 points if the information concerned repeated or extended events or partial recollection. 0 marks were awarded for speculative answers or supposition.

\section{Assessment of Autonoetic Consciousness}

Participants' recollection of ABMs were examined across the following behavioral domains posited to be important for the subjective recollective experience (Addis et al., 2004; Irish et al., 2008; Piolino et al., 2006); Vividness of Imagery Evoked, Viewer Perspective, Continuity of Accompanying Imagery, Frequency of Covert Rehearsal, Frequency of Overt Rehearsal, Emotional Re-experiencing rating, Emotional Valence, Overall Re-experiencing rating. Participants were also asked to confer a global judgment of "Reliving" or "Looking Back" on each memory. The EAMI paradigm was shortened for the present study by requiring participants to recall one memory per epoch as opposed to previous studies where three memories per epoch were assessed (Irish et al., in press). 


\section{Experimental Investigation of Context-Dependent Memory}

To measure acquisition of a recent event and the potential facilitative effect of context-dependent memory, we investigated participants' recall of a cognitive screening session carried out in the memory clinic 1 week previously. The objective details of this screening session were recorded by the experimenter across each of the phenomenological categories on the EAMI, providing an independently verified account of the event for subsequent cross-checking of participants' recall. Testing took place in the same room, at the same time of day 1 week later, and this memory for "Last Week" was scored according to the standard EAMI 7-point protocol. The subjective recollective experience for this event was also probed, as above. This permitted the investigation of a veridical memory that was recalled in the same spatiotemporal context, controlling for retention interval, age of participants, and emotional tone.

\section{Statistical Analyses}

To determine between-group differences across the cognitive screening variables, multivariate analyses of variance (MANOVA) were run, with nonparametric tests (Mann-Whitney $U$ ) used for variables with non-normal distributions. For personal semantics and event details, the key factors of interest were the between-group and withingroup differences across life epochs on the EAMI. Repeated measures ANOVAs were used to investigate the factors of group (EC, aMCI) and paired $t$ tests with Bonferroni corrections ( $\alpha /$ number of comparisons) were used to investigate epoch effects. The mean differences between groups for PS and Event recall were calculated with 95\% Confidence Intervals.

To investigate group differences in the subjective recollective experience, $\chi^{2}$ statistics based on the frequency patterns in the variables were run. To determine the predictive efficacy of the behavioral markers for autonoetic consciousness, all participants' memories (EC and aMCI), across all life epochs $(n=204)$ were included in a Backwards Wald Stepwise Binary Logistic regression analysis, with "looking back" set as the reference category for the dependent variable. The resulting significant Exponential Beta values were converted to odds ratios for reliving judgments by inversion i.e. Odds Ratio $=1 / \operatorname{Exp}(B)$. As an additional check, a binary logistic regression was run using gender as a predictive variable for reliving. To explore possible relationships between tests of executive function and reliving judgments, specific correlational analyses were conducted using Pearson's R.

\section{RESULTS}

\section{Demographics}

Elderly controls were recruited from an Active Retirement Association and ranged in age from 69 to 86 years (age: mean $\pm S D=76.0 \pm 4.3)$ with an average of 14.0 years $( \pm 3.1)$ in formal education (range: 11-21 years) (see Table 1). aMCI participants ranged in age from 62 to 88 years (age: $71.8 \pm$ 6.8 years) and spent an average of 13.8 years ( \pm 4.7 years) in formal education (range: 7.5-25 years). There was a significant gender difference between the groups $\left(\chi^{2}(33)=5.673\right.$; $p=.17)$ with a female:male ratio of $14: 4$ in the EC group compared with 6:10 in the aMCI group. The groups were matched for years in education $(p=.592)$; however, the aMCI group was on average 4.2 years younger than the EC group $(F(1,32)=4.658 ; p=.039)$.

\section{Recall of Personal Semantic Information}

Figure 1 shows the overall performance of EC and aMCI participants on the personal semantic subscale of the EAMI. A repeated measures ANOVA revealed a significant main effect for epoch $(F(4,128)=4.189 ; p=.003)$ and an epoch $\times$ group interaction $(F(4,128)=4.957 ; p=.001)$ with aMCI recall most compromised in Later Adulthood. A significant group effect was found $(F(1,32=27.963 ; p<.0001)$, with ECs performing at higher levels than aMCI participants across all epochs except Childhood $(p=.627)$ and Early Adulthood $(p=.066$ ) (Middle Adulthood Mean Difference $-2.22[95 \%$ CI $=-3.39,-1.04]$; Later Adulthood Mean Difference -2.26 [95\% CI $=-3.30,-1.14]$; Recent Period Mean Difference -1.62 [95\% CI $=-2.47,-0.78]$ ).

ECs showed better recall in more recent epochs compared with Childhood $(t=-3.183 ; p=.005)$, with best personal semantic recall demonstrated in Middle Adulthood compared with both Childhood $(\mathrm{t}=-5.682 ; p<.0001)$ and Recent period $(t=3.154 ; p=.006)$. For aMCI participants, there were no significant differences in personal semantic recall across life epochs.

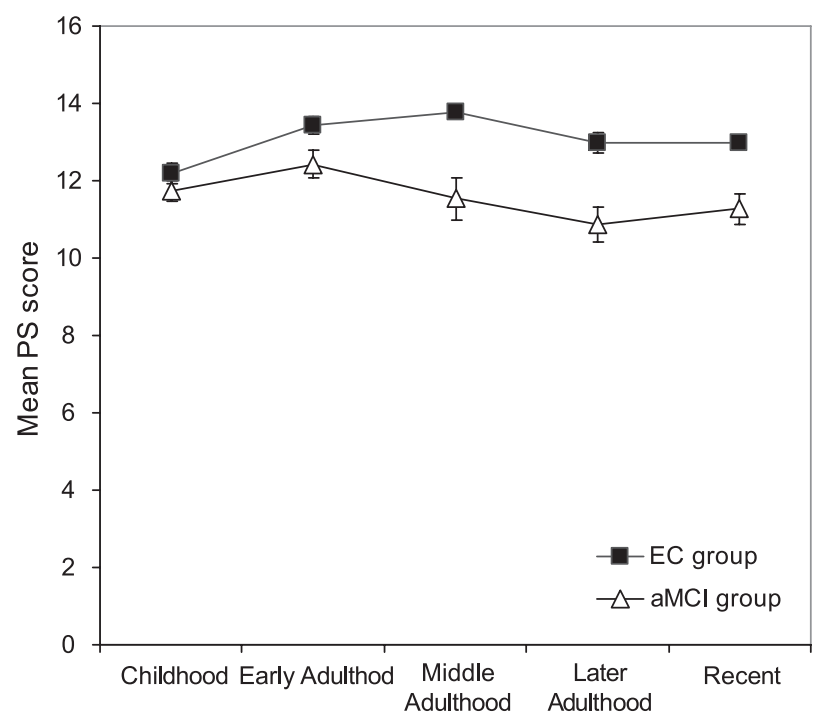

Fig. 1. Personal Semantic recall across the lifespan for EC and aMCI individuals. 


\section{Recall of Contextual Details for Autobiographical Events}

Figure 2 shows the recall of contextual details across life epochs on the EAMI for EC and aMCI participants. A repeated measures ANOVA revealed a significant main effect for epoch $(F(5,160)=8.208 ; p<.0001)$, and an epoch*group interaction $(F(5,160)=19.404 ; p=.001)$ with recall of contextual details most compromised in Middle Adulthood in aMCI. A significant group effect was found $(F(1,32)=$ 69.211; $p<.0001$ ), with ECs recalling significantly higher levels of contextual details than aMCI participants across all epochs. This group effect was most pronounced for the Middle Adulthood $(F(1,32)=54.823 ; p<.0001)$ and "Last Week" epochs $(F(1,32)=57.468 ; p<.0001)$.

There were no significant within-group differences across epochs for aMCI participants for recall of contextual details. However, an epoch effect was found in the EC group, with the greatest level of contextual detail recalled in the "Last Week" period in comparison with all other epochs (Childhood: $t=-6.860 ; p<.0001$; Early Adulthood: $t=-4.761$; $p<.0001$; Middle Adulthood: $t=-3.436 ; p=.003$; Later Adulthood: $t=-4.267 ; p=.001$; Recent Period: $t=-5.067$; $p<.0001)$. A univariate ANOVA revealed there was no main effect for gender ( $p=.237)$, however a group*gender interaction was found $(F(1,200)=7.170 ; p=.008)$ with female aMCI participants exhibiting the lowest average level of contextual details (Mean details score $=2.96$ ) in comparison with all other groups (male aMCI $=3.67$ ). There was no gender difference within the EC group (Mean details scores: male $\mathrm{EC}=5.04$; female $\mathrm{EC}=5.31 ; p=.304$ ) for recall of details.

\section{Incidence of Reliving during Event Recall}

The $\chi^{2}$ tests revealed that there was no significant difference in the total number of memories relived between the EC

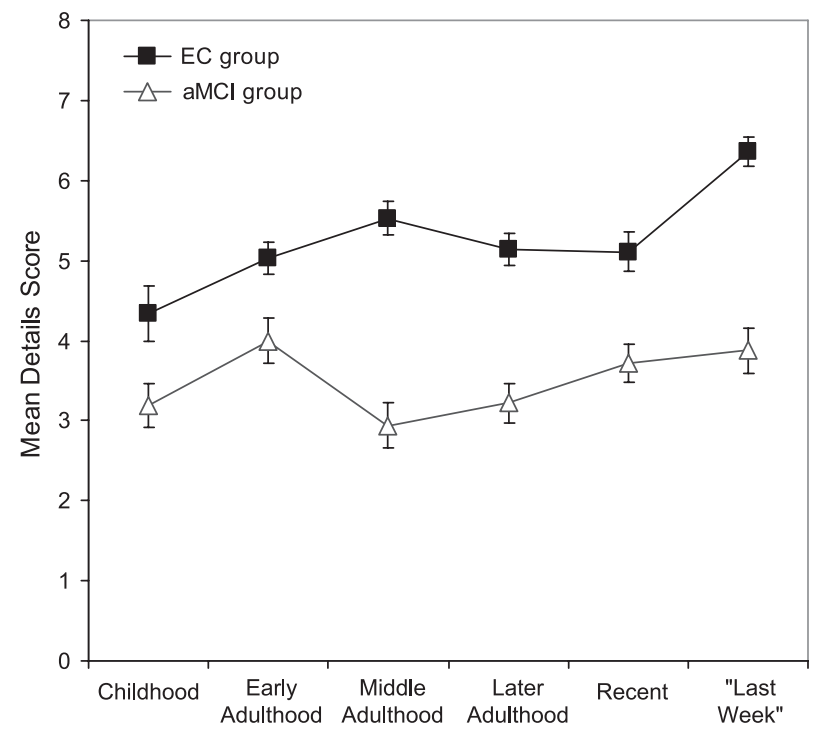

Fig. 2. Recall of contextual details across the lifespan for EC and aMCI individuals including additional "Last week" memory. (Maximum score is 7 points.)
$(18.5 \%)$ and aMCI group $(11.5 \%)\left(\chi^{2}(1)=1.392 ; p=.238\right)$ (see Figure 3).

There were no significant differences in reliving judgments across life epochs for aMCI participants $\left(\chi^{2}(5)=\right.$ $3.429 ; p=.634$ ), with the majority of memories recalled via noetic "looking back" judgments. Similarly, there were no epoch effects for reliving judgments in the EC group $\left(\chi^{2}(5)=\right.$ $7.118 ; p=.212)$. However, there was a suggestion of a recency effect with approximately one-third of EC relived memories ( $6.5 \%$ of total memories) occurring in the Recent period.

\section{Group Differences and Epoch Effects in the Recollective Experience}

Table 2 demonstrates that the majority of behavioral markers on the autonoetic subscale of the EAMI discriminated between the EC and aMCI groups, except for the global judgment of reliving, emotional valence and overall re-experiencing ratings. The $\chi^{2}$ analyses collapsed across both participant groups revealed there was no gender effect for any of the recollective variables $(p>.10)$. We also ran a logistic regression using gender as a predictive variable for reliving, and this was not found to exert a significant predictive effect $(p=.285)$. Furthermore, $\chi^{2}$ analyses revealed that there was no difference between single-domain $(n=3)$ and multi-domain aMCI cases $(n=13)$ across any of the recollective variables $(p>.05)$.

Recency effects were observed for ECs on many of the autonoetic markers, with memories from the Recent period most often rated as "very vivid" (55.6\%) in comparison with Childhood $(27.8 \%)\left(\chi^{2}(25)=55.803 ; p<.0001\right)$ and more often visualized as continuous "video clips" (Recent: $50 \%$, Last week: $61.1 \%)\left(\chi^{2}(25)=37.619 ; p=.050\right)$. There was no difference across epochs for viewer perspectives $\left(\chi^{2}(15)=17.067\right.$;

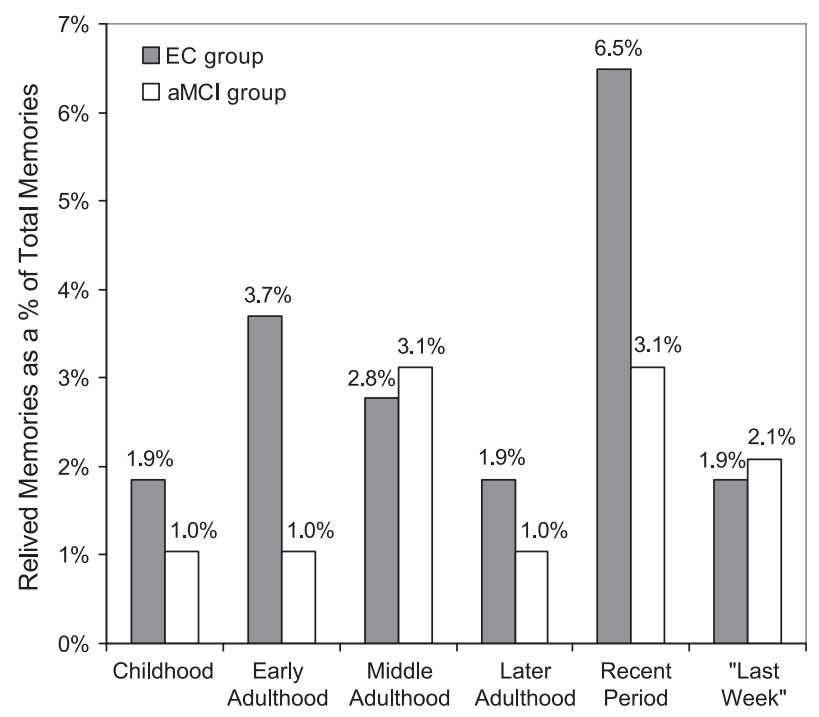

Fig. 3. Distribution of reliving judgments as a percentage of the total number of memories recalled on the EAMI for EC and aMCI participants. 
Table 2. Group differences across each autonoetic marker on the EAMI, as revealed by the $\chi^{2}$ statistic

\begin{tabular}{|c|c|c|c|c|}
\hline Marker ${ }^{\mathrm{a}}$ & Rating & EC group & aMCI group & Group effect \\
\hline Reliving & & $18.5 \%$ & $11.5 \%$ & $\mathrm{n} / \mathrm{s}$ \\
\hline \multirow[t]{2}{*}{ Vividness } & "Very Vivid" & $25.0 \%$ & $4.2 \%$ & $* * *$ \\
\hline & "Quite Vague/Vague" & $5.6 \%$ & $18.8 \%$ & \\
\hline \multirow[t]{2}{*}{ Viewer Perspective } & "Own eyes" & $36.1 \%$ & $12.5 \%$ & $* * *$ \\
\hline & "Off-tangent" imagery & $0.9 \%$ & $21.9 \%$ & \\
\hline \multirow{2}{*}{ Continuity of Imagery } & "Video"/"Video clips" & $46.3 \%$ & $9.2 \%$ & $* * *$ \\
\hline & "One snapshot"/“Hazy image" & $13.9 \%$ & $50.0 \%$ & \\
\hline \multirow[t]{2}{*}{ Emotional Re-experiencing } & “75-100\% Re-experiencing” & $24.0 \%$ & $10.4 \%$ & $*$ \\
\hline & "0-25\% Re-experiencing" & $52.8 \%$ & $70.8 \%$ & \\
\hline Emotional Valence & Positive & $57.3 \%$ & $61.1 \%$ & $\mathrm{n} / \mathrm{s}$ \\
\hline Covert Rehearsal & "Rarely/Never Think about it" & $34.3 \%$ & $65.6 \%$ & $* * *$ \\
\hline Overt Rehearsal & "Never Think about it" & $8.3 \%$ & $33.3 \%$ & $* * *$ \\
\hline \multirow[t]{2}{*}{ Overall Re-experiencing } & "0\% Re-experiencing" & $56.5 \%$ & $63.5 \%$ & $\mathrm{n} / \mathrm{s}$ \\
\hline & "75-100\% Re-experiencing" & $11.1 \%$ & $8.4 \%$ & \\
\hline
\end{tabular}

Note. Those subcategories of each marker which showed the greatest degree of discrimination between groups have been presented. The full range of subcategories in each autonoetic marker are described by Irish et al. (in press). $*^{* *} p<.0001 ; * p<.05$. $\mathrm{n} / \mathrm{s}=$ nonsignificant .

$p=.315)$, however remote memories tended to be viewed more often from a third person perspective by ECs $(66.7 \%)$. Recent memories were emotionally re-experienced to a greater degree by ECs (50\% level - $27.8 \%$ of memories, $75 \%$ level $-50 \%$ of memories) in comparison with Childhood $(50 \%$ level $-0 \% ; 75 \%$ level $-16.7 \%)\left(\chi^{2}(20)=39.451 ; p=\right.$ .006). Conversely, Childhood and Early Adulthood memories were more often rated at lower levels of emotional re-experiencing ( $0 \%$ level: $72.2 \%$ and $38.9 \%$, respectively). Memories in more recent epochs tended to be rated by ECs as thought about $\left(\chi^{2}(15)=30.862 ; p=.009\right)$ and discussed $\left(\chi^{2}(15)=\right.$ $43.323 ; p<.0001)$ more frequently than those in more remote epochs. In contrast, recency effects were not evident in the aMCI group, with comparable ratings provided irrespective of time period.

\section{Predictive Efficacy of Behavioral Markers for the Recollective Experience}

Figure 4 shows the binary logistic regression results for all participants' memories combined (EC and aMCI; $n=204$ ), with those predictors of reliving presented in ascending order according to the magnitude of their odds ratios. The best predictors of autonoetic reliving during ABM retrieval related to the visual imagery evoked during recall; the "reallife" playing out of the memory akin to a video, followed by the viewing of the memory as video clips, and the using of an egocentric "own eyes" perspective. A linear regression using reliving as the default variable and the tests of executive function as predictive variables was run for all participants combined (EC and aMCI). This indicated that none of the executive tasks exerted a significant predictive effect for judgments of reliving $(p>.05)$. Correlational analyses using Pearson's R did not reveal any significant relationship between performance on the executive tests and reliving judg- ments for ECs ( $r$ from -0.125 to 0.279 , all $p>.2$ ). However, in the aMCI group, total spatial span $(r=-.612 ; p=.012)$ and category fluency $(r=-.537 ; p=.032)$ exhibited significant inverse relationships with reliving, with lower scores on these tests associated with noetic "looking back" judgments. Correlational analyses failed to find any significant relationship between executive tests and recall of personal semantics or episodic details ( $r$ from -.457 to .262 , all $p>.05$ ).

\section{DISCUSSION}

The objective of this study was to investigate ABM in amnestic MCI, with a view to identifying potential deficits

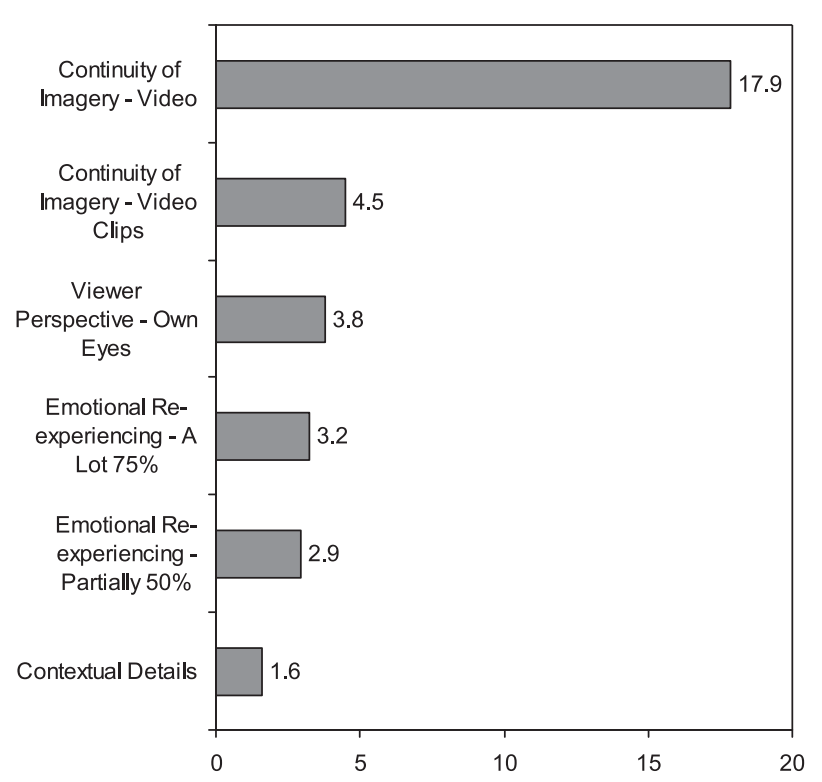

Fig. 4. Autonoetic marker odds ratios ranked in cascading order according to predictive efficacy for reliving judgments. 
across three domains, namely (i) personal semantics, (ii) event details, and (iii) the recollective experience. Key findings of this study concern the compromised recall of both personal semantic and event details in aMCI, albeit at differential levels. aMCI participants did not benefit from the provision of covert cues in the "Last Week" memory condition, despite this memory being tested in the same spatiotemporal context. Whereas there was no group dissociation for frequency of reliving judgments, significant impairments were evident in the aMCI group across multiple recollective markers notably vividness, viewer perspective, continuity of imagery, and emotional re-experiencing. We discuss the three axes of ABM and the importance of visual imagery and emotional re-experiencing as factors inextricably linked to the recollective experience.

In contrast with previous studies (Murphy et al., 2008), we have demonstrated that personal semantic memory is compromised in aMCI in comparison with healthy elderly controls. Personal semantic information can often be facilitated in older adults (Levine et al., 2002), proving relatively resistant to the effects of age and retention period (Piolino et al., 2002), and the high scores demonstrated by our EC group support Bahrick's (1984) conception of a permanent memory store of personal semantics. Our aMCI cohort showed an impaired capacity to retrieve personal semantics from the Middle Adulthood period onward, with a significant group by epoch interaction stemming from their particularly impaired recall in the Later Adulthood period. This diverges somewhat from the findings of Leyhe et al. (2009) of impaired personal semantic recall in aMCI confined to the Recent period using the AMI. Methodological differences may account for such temporal discrepancies, as the AMI probes recall across three time periods in contrast to the five epochs of the EAMI. Of note, however, is the finding that using a new ABM protocol, we have demonstrated significant impairments for personal semantics extending beyond the Recent period in aMCI.

For event recall, aMCI participants exhibited impairments across all epochs, recalling extended personal accounts divested of rich contextual details, with the greatest group difference evident in the "Last Week" epoch (Mean Difference = 2.49). This was mediated by the near-perfect recall of this event by the EC group (Mean score: 6.36), suggesting the facilitative effect of recall occurring within the same spatiotemporal context. Conversely, details that were most disrupted for aMCI participants during this epoch were those relating to spatiotemporal specificity and sensory-perceptual details. This is of particular significance considering such details were covertly provided during the testing of the "Last Week" memory, yet the aMCI group were unable to assimilate these cues into a cohesive account of the event. Anderson, Ebert, Jennings, Grady, Cabeza, and Graham (2008) used a word-learning task to show that aMCI participants exhibit a selective disruption for recollection of items in their context. Here we have demonstrated a deficit in context-dependent memory within a naturalistic setting, replicating the subtle lapses often displayed in everyday memory.
Autonoetic reliving was low for both participant groups $(\mathrm{EC}=18.5 \%$; aMC $\mathrm{I}=11.5 \%)$, in keeping with previous studies (Irish et al., in press), possibly indicating a floor effect. Counter to our original hypothesis, there was no significant group difference for frequency of reliving judgments, however the small sample size may have obscured potential differences. By sampling one memory per epoch, we may have constrained the recollective process such that participants selected the most accessible memory consistent with their self-schema for each epoch (Conway, 2001) rather than the most emotive experience. In a previous study, we demonstrated the predictive efficacy of negatively valenced emotionally arousing events for the recollective experience, however this effect was elicited following probing of three memories per epoch (Irish et al., in press). Our present participants recalled a preponderance of positive events $(\mathrm{EC}=61.1 \%$; $\mathrm{MCI}=57.3 \%)$, consistent with a diminished emphasis on negative emotions with age (Carstensen, Fung, \& Charles, 2003; Hedden and Gabrieli, 2004). It is possible that, had participants been asked to recall additional events for each life epoch, differences in the frequency of memories relived between the groups may have been observed.

Whereas reliving judgments did not discriminate between the groups, elements of the recollective experience were noticeably disrupted in the aMCI group. For vividness and viewer perspective, aMCI participants tended to generate off-tangent visual imagery unrelated to the event being discussed. Similarly, the continuity of visual imagery in the aMCI group was fragmented and degraded in quality, with memories more often resembling static snapshots or hazy images. These findings suggest that concomitant with the burgeoning anterograde episodic impairments in aMCI is the compromised capacity to generate vivid, self-referential, and "real-life" visual imagery during ABM recall. Although visual imagery generation does not necessarily guarantee memory accuracy, a large corpus of research contends that the experience of recollecting an ABM coincides with the successful retrieval of a visual image (Brewer, 1996; Gardini, Cornoldi, De Beni, \& Venneri, 2006). This is borne out in the present study where viewing memories as "real life" videos exerted the strongest predictive power for reliving past memories. Our study also reinforces the importance of emotional re-experiencing as a predictor for the sense of recollection, with aMCI participants re-experiencing the original emotion of events significantly less than controls. Given such compromised visual imagery and diminished levels of emotional re-experiencing, the failure to find deficits on the global judgment of reliving in aMCI is surprising, although a larger pool of memories may have revealed significant group differences. The ability to navigate successfully within subjective time is likely mediated by a complex frontotemporal system (Moulin, Conway, Thompson, James, \& Jones, 2005) and the impoverished visual and emotional re-experiencing of past events in aMCI may stem in part from their executive deficits. The association between lower scores on executive function tasks (spatial span and category fluency) and "looking back" judgments suggests the importance of the frontal 
lobes during the recollective experience. It is possible that further relationships among the variables were obscured by our small sample sizes.

Consistent with previous reports (Irish et al., in press; Piolino et al., 2006; Piolino, Giffard-Quillon, Desgranges, Chetelat, Baron, \& Eustache, 2004), elderly controls demonstrated the highest frequency of reliving in the Recent period (38.9\% of Recent memories), with these memories viewed from the "own eyes" perspective, resembling real-life "video clips", and accompanied with high levels of emotional reexperiencing. Interestingly, memories from the "Last Week" epoch did not instill a sense of recollection in the EC group $(1.9 \%)$ despite the context-dependent memory effect. This runs counter to the proposition that it is the details and the details alone that mediate episodic retrieval (Moscovitch et al., 1999; Piolino et al., 2002). This epoch represents something of an anomaly where the constructs of episodic and semantic memory are concerned. If we adopt the criteria of Wheeler, Stuss, and Tulving (1997), the dearth of reliving judgments for these memories render them "not episodic" and therefore semantic memories. Whereas we cannot label the "Last Week" memories as strictly episodic, it is also clear that these memories do not qualify as semanticized accounts given their short retention interval. If memories that are relived are qualitatively distinct from all other memories, we would expect to see marked differences between the "Last Week" and Recent memories. However, direct comparison of these epochs revealed similarities across all recollective markers except that of emotional re-experiencing. In the "Last Week" condition, participants were instructed to recall a specific, time-locked event that appeared not to carry any significant emotional import for participants (EC Neutral valence $38.9 \%$ ) in comparison with self-selected personally significant Recent memories (EC Neutral valence: 5.6\%). This suggests that, whereas participants are not reliving the memory, they are still re-experiencing certain elements of it (e.g., viewer perspective, continuity of imagery), pointing to the critical feature of emotional connectedness for a reliving experience.

We suggest that the degree of interaction between the episodic and semantic memory systems may be considerably greater than previously assumed. Furthermore, the recollective experience may be reconceived along a continuum, as opposed to a dichotomous, all-or-nothing phenomenon. We propose a new taxonomy of ABM (see Figure 5), in which episodic memory is dissociated into the subcomponents of "reliving" and "re-experiencing," representing emotional episodic memory and nonemotional episodic memory, respectively. To relive a memory would rely on the highest level of autonoetic consciousness, of which the critical feature is the reinstantiation of the original emotional experience. Accordingly, it would be possible to re-experience elements of a past event without this culminating in the full "reliving" of that event. The degree of engagement of the episodic memory system would be contingent on the emotional disposition of the individual at the time of retrieval, and as such could be influenced by the nature of the task or modified by manipulation of one's emotional state. If this is possible, the relationship between episodic and semantic memory systems is not bidirectional, as one could not recall a memory that has been semanticized over many years in an emotionally rich episodic manner. We argue that it is possible to recall an event-based memory in one of three ways; as (i) an emotionally-laden episodic memory that is relived, (ii) an episodic memory with elements that are re-experienced, or (iii) as a semantic memory that may or may not contain a high level of contextual detail. Accordingly, the memory for "Last Week" would reflect engagement of the "Nonemotional episodic memory" system in Figure 5. Of interest, we have demonstrated the compromised capacity to successfully retrieve events within this branch of episodic memory in aMCI and the unraveling of the subjective recollective experience. Limitations of the study include the relatively small sample size from which we draw our conclusions, as well as the uneven gender distribution between the participant groups, an unfortunate consequence of convenience sampling. Although post hoc tests did not reveal a systematic gender effect for ABM recall and the recollective experience, we must acknowledge this as a potential bias given extant literature highlighting gender discrepancies in the nature of ABM recall for young (Irish et al., 2008) and elderly participants (Pillemer, Wink, DiDonato, \& Sanborn, 2003). A direct comparison between healthy controls, aMCI and individuals with mild $\mathrm{AD}$ might prove revelatory in highlighting to what extent aMCI participants exhibit a prodromal $\mathrm{AD}$ profile or one that is more similar to healthy aging, and

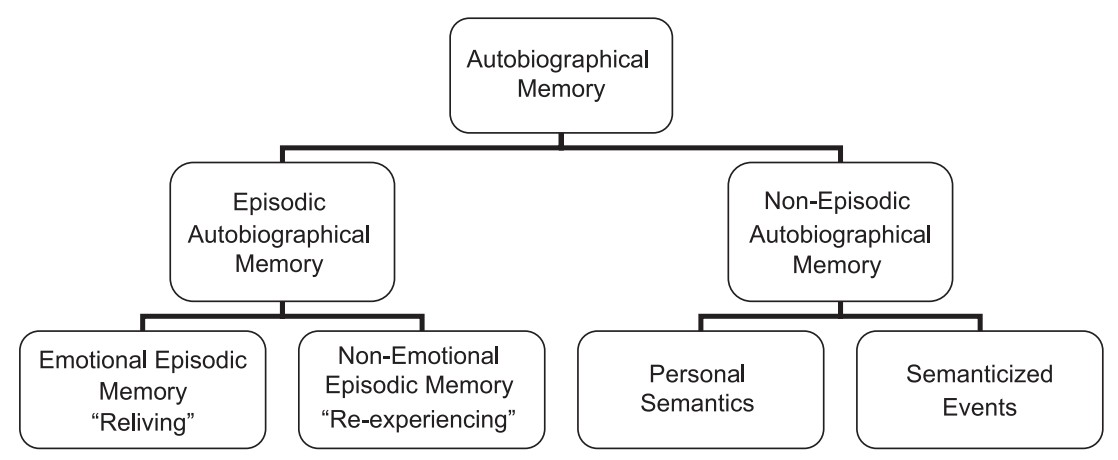

Fig. 5. A modified view of the episodic/semantic distinction within autobiographical memory. 
this comparison would be further complemented by neuroimaging data.

\section{CONCLUSION}

This study has revealed deficits in aMCI across three axes of ABM; namely personal semantics, episodic contextual details, and reliving via autonoetic consciousness. Whereas the global capacity for reliving appears undiminished in aMCI, closer inspection reveals a breakdown in the phenomenological and experiential aspects critical to engaging the episodic autobiographical memory system, particularly that of visual imagery. Our inclusion of a veridical memory for "Last Week" pointed to emotional re-experiencing as a critical mediator of reliving. We propose a new taxonomy of episodic autobiographical memory to incorporate emotional episodic memories ("reliving") and nonemotional episodic memories ("re-experiencing"), the latter of which is disrupted in aMCI and warrants further investigation as a potential predictor of conversion to $\mathrm{AD}$, particularly within longitudinal studies.

\section{ACKNOWLEDGEMENTS}

We thank the participants and their families who cooperated in this study, and the nursing and medical staff of Mercer's Institute for Research on Aging (MIRA), St. James's Hospital, Dublin, for their assistance in patient recruitment. M.I. thanks the Higher Education Authority and MIRA for financial support. The authors report no conflict of interest.

\section{REFERENCES}

Addis, D.R., Moscovitch, M., Crawley, A.P., \& McAndrews, M.P. (2004). Recollective qualities modulate hippocampal activation during autobiographical memory retrieval. Hippocampus, 14, 752-762.

Aggarwal, N.T., Wilson, R.S., Beck, T.L., Bienias, J.L., \& Bennett, D.A. (2005). Mild cognitive impairment in different functional domains and incident Alzheimer's disease. Journal of Neurology, Neurosurgery, and Psychiatry, 76, 1479-1484.

Anderson, N.D., Ebert, P.L., Jennings, J.M., Grady, C.L., Cabeza, R., \& Graham, S.J. (2008). Recollection- and familiarity-based memory in healthy aging and amnestic Mild Cognitive Impairment. Neuropsychology, 22, 177-187.

Arnaiz, E., \& Almkvist, O. (2003). Neuropsychological features of mild cognitive impairment and preclinical Alzheimer's disease. Acta Neurologica Scandinavica, 107(Suppl. 179):34-41.

Bahrick, H.P. (1984). Semantic memory content in permastore: Fifty years of memory for Spanish learned in school. Journal of Experimental Psychology: General, 113, 1-29.

Brewer, W.F. (1996). What is recollective memory? In D.C. Rubin (Ed.), Remembering our past: Studies in Autobiographical Memory (pp. 19-66). New York: Cambridge University Press.

Carstensen, L.L., Fung, H.H., \& Charles, S.T. (2003). Socioemotional selectivity theory and the regulation of emotion in the second half of life. Motivation and Emotion, 27, 103-123.

Conway, M.A. (2001). Sensory-perceptual episodic memory and its context: Autobiographical memory. Philosophical Transactions of the Royal Society of London B Biological Sciences, 356, 1375-1384.
Conway, M.A., Pleydell-Pearce, C.W., Whitecross, S., \& Sharpe, H. (2002). Brain imaging autobiographical memory. Psychology of Learning and Motivation, 41, 229-264.

Delacourte, A., David, J.P., Sergeant, N., Bueé, L., Wattez, A., Vermersch, P., et al. (1999). The biochemical pathway of neurofibrillary degeneration in aging and Alzheimer's disease. Neurology, 52, 1158-1165.

Fischer, P., Jungwirth, S., Zehetmayer, S., Weissgram, S., Hoenigschnabl, S., Gelpi, E., et al. (2007). Conversion from subtypes of mild cognitive impairment to Alzheimer dementia. Neurology, 68, 288-291.

Folstein, M.F., Folstein, S.E., \& McHugh, P.R. (1975). Mini-mental state: A practical method for grading the cognitive state of patients for the clinician. Journal of Psychiatry Research, 12, 189-198.

Ganguli, M., Dodge, H.H., Shen, C., \& DeKosky, S.T. (2004). Mild cognitive impairment, amnestic type: An epidemiologic study. Neurology, 63, 115-121.

Gardini, S., Cornoldi, C., De Beni, R., \& Venneri, A. (2006). Left mediotemporal structures mediate the retrieval of episodic autobiographical mental images. Neuroimage, 30, 645-655.

Gauthier, S., Reisberg, B., Zaudig, M., Petersen, R.C., Ritchie, K., Broich, K., et al. (2006). Mild cognitive impairment. Lancet, 367, 1262-1270.

Hedden, T., \& Gabrieli, J.D.E. (2004). Insights into the aging mind: A view from cognitive neuroscience. Nature Reviews Neuroscience, 5, 87-96.

Herrmann, N., Mittman, N., Silver, I.L., Shulman, K.I., Busto, U.A., Shear, N.H., et al. (1996). A validation study of the Geriatric Depression Scale short form. International Journal of Geriatric Psychiatry, 11, 457-460.

Irish, M., Lawlor, B.A., O’Mara, S.M., \& Coen, R.F. (2008). Assessment of behavioural markers of autonoetic consciousness during episodic autobiographical memory retrieval: A preliminary analysis. Behavioural Neurology, 18, 1-4.

Irish, M., Lawlor, B.A., O’Mara, S.M., \& Coen, R.F. (in press). Impaired capacity for autonoetic reliving during autobiographical event recall in mild Alzheimer's disease. Cortex.

Kopelman, M.D., Wilson, B., \& Baddeley, A.D. (1989). The autobiographical memory interview: A new assessment of autobiographical and personal semantic memory in amnesic patients. Journal of Clinical and Experimental Neuropsychology, 11, 724-744.

Lawton, M.P., \& Brody, E.M. (1969). Assessment of older people: Self-maintaining and instrumental activities of daily living. Gerontologist, 9, 179-186.

Levine, B., Svoboda, E., Hay, J.F., Winocur, G., \& Moscovitch, M. (2002). Aging and autobiographical memory: Dissociating episodic from semantic retrieval. Psychology and Aging, 17, 677-689.

Leyhe, T., Muller, S., Milian, M., Eschweiler, G.W., \& Saur, R. (2009). Impairment of episodic and semantic autobiographical memory in patients with mild cognitive impairment and early Alzheimer's disease. Neuropsychologia, 47, 2464-2469.

Luis, C.A., Barker, W.W., Loewenstein, D.A., Crum, T.A., Rogaeva, E., Kawarai, T., et al. (2004). Conversion to dementia among two groups with cognitive impairment: A preliminary report. Dementia and Geriatric Cognitive Disorders, 18, 307-313.

Manos, P.J., \& Wu, R. (1994). The ten point clock test: A quick screen and grading method for cognitive impairment in medical and surgical patients. International Journal of Psychiatry Medicine, 24, 229-244. 
Masdeu, J.C., Zubieta, J.L., \& Arbizu, J. (2005). Neuroimaging as a marker of the onset and progression of Alzheimer's disease. Journal of the Neurological Sciences, 236, 55-64.

Moscovitch, M., Yaschyshyn, T., Ziegler, M., \& Nadel, L. (1999). Remote episodic memory and retrograde amnesia: Was Endel Tulving right all along? In E. Tulving (Ed.), Memory, consciousness and the brain: The Tallinn conference. New York: Psychology Press.

Moulin, C.J.A., Conway, M.A., Thompson, R.G., James, N., \& Jones, R.W. (2005). Disordered memory awareness: Recollective confabulation in two cases of persistent déjà vecu. Neuropsychologia, 43, 1362-1378.

Murphy, K.J., Troyer, A.K., Levine, B., \& Moscovitch, M. (2008). Episodic, but not semantic, autobiographical memory is reduced in amnestic mild cognitive impairment. Neuropsychologia, 46, 3116-3123.

Nelson, H.E., \& Willison, J. (1991). National adult reading test (NART): Test manual. Windsor: NFER-Nelson.

Pennanen, C., Kivipelto, M., Tuomainen, S., Hartikainen, P., Hanninen, T., Laakso, M.P., et al. (2004). Hippocampus and entorhinal cortex in mild cognitive impairment and early AD. Neurobiology of Aging, 25, 303-310.

Perri, R., Carlesimo, G.A., Serra, L., \& Caltagirone, C. (2005) Characterization of memory profile in subjects with amnestic mild cognitive impairment. Journal of Clinical and Experimental Neuropsychology, 27, 1033-1055.

Petersen, R.C., Doody, R., Kurz, A., Mohs, R.C., Morris, J.C., Rabins, P.V., et al. (2001). Current Concepts in Mild Cognitive Impairment. Archives of Neurology, 58, 1985-1992.

Petersen, R.C., \& Morris, J.C. (2005). Mild cognitive impairment as a clinical entity and treatment target. Archives of Neurology, 62, 1160-1163.

Piefke, M., Weiss, P.H., Zilles, K., Markowitsch, H.J., \& Fink, G.R. (2003). Differential remoteness and emotional tone modulate the neural correlates of autobiographical memory. Brain, 126, 650-668.

Pike, K.E., \& Savage, G. (2008). Memory profiling in mild cognitive impairment: Can we determine risk for Alzheimer's disease? Journal of Neuropsychology, 2, 361-372.

Pillemer, D.B., Wink, P., DiDonato, T.E., \& Sanborn, R.L. (2003). Gender differences in autobiographical memory styles of older adults. Memory, 11, 525-532.

Piolino, P., Desgranges, B., Belliard, S., Matuszewski, V., Lalevée, C., de la Sayette, V., et al. (2003). Autobiographical memory and autonoetic consciousness: Triple dissociation in neurodegenerative diseases. Brain, 126, 2203-2219.

Piolino, P., Desgranges, B., Benali, K., \& Eustache, F. (2002). Episodic and semantic remote autobiographical memory in aging. Memory, 4, 239-257.

Piolino, P., Desgranges, B., Clarys, D., Guillery-Girard, B., Taconnat, L., Isingrini, M., et al. (2006). Autobiographical memory, autonoetic consciousness and self-perspective in aging. Psychology and Aging, 21, 510-525.

Piolino, P., Giffard-Quillon, G., Desgranges, B., Chetelat, G., Baron, J.C., \& Eustache, F. (2004). Re-experiencing old memories via hippocampus: A PET study of autobiographical memory. Neuroimage, 22, 1371-1383.

Portet, F., Ousset, P.J., Visser, P.J., Frisoni, G.B., Nobili, F., Scheltens, P., et al. (2006). Mild cognitive impairment (MCI) in medical practice: A critical review of the concept and new diagnostic procedure. Report of the MCI Working Group of the European Consortium on Alzheimer's disease. Journal of Neurological Neurosurgical Psychiatry, 77, 714-718.

Rauchs, G., Piolino, P., Mézenge, F., Landeau, B., Lalevée, C., Pélerin, A., et al. (2007). Autonoetic consciousness in Alzheimer's disease: Neuropsychological and PET findings using an episodic learning and recognition task. Neurobiology of Aging, 28, 1410-1420.

Reitan, R.M. (1958). Validity of the trail making test as an indicator of organic brain damage. Perceptual Motor Skills, 8, 271-276.

Rubin, D.C. (2005). A basic-systems approach to autobiographical memory. Current Directions in Psychological Science, 14, 79-83.

Sheikh, J.I., \& Yesavage, J.A. (1986). The geriatric depression scale (GDS) recent evidence and development of a shorter version. Clinical gerontology: A guide to assessment and intervention (pp. 165-173). NY: The Haworth Press; 1986.

Tabert, M.H., Manly, J.J., Liu, X., Pelton, G.H., Rosenblum, S., Jacobs, M., et al. (2006). Neuropsychological prediction of conversion to Alzheimer's disease in patients with mild cognitive impairment. Archives of General Psychiatry, 63, 916-924.

Trenerry, M.R., Crosson, B., DeBoe, J., \& Leber, W.R. (1989). Stroop neuropsychological screening test. Odessa FL: Psychological Assessment Resources.

Tulving, E. (2001). Episodic memory and common sense: How far apart? Philosophical Transactions of the Royal Society of London, B: Biological Sciences, 356, 1505-1515.

Visser, P.J., Scheltens, P., \& Verhey, F.R.J. (2005). Do MCI criteria in drug trials accurately identify subjects with predementia Alzheimer's disease? Journal of Neurology, Neurosurgery, and Psychiatry, 76, 1348-1354.

Wechsler, D. (1997). Wechsler memory scale (III). San Antonio, TX: The Psychological Corporation.

Wheeler, M.A., Stuss, D.T., \& Tulving, E. (1997). Toward a theory of episodic memory: The frontal lobes and autonoetic consciousness. Psychological Bulletin, 121, 331-354.

Winblad, B., Palmer, K., Kivipelto, M., Jelic, V., Fratiglioni, L., Wahlund, L.O., et al. (2004). Mild cognitive impairment beyond controversies, toward a consensus: Report of the International Working Group on Mild Cognitive Impairment. Journal of Internal Medicine, 256, 240-246. 